\title{
Inversión en un estudiante universitario
}

\author{
Br. Eliasid Anelores Delgado, Bra. Brenda Carolina Toledo Narváez, \\ Br. Rony Kadir Vargas Narváez.
}

Facultad Regional Multidisciplinaria- Carazo. ${ }^{1}$

Recibido: 24 de abril 2013- Aprobado 28 de octubre 2013.

RESUMEN

El presente trabajo está centrado en determinar y analizar los costos incurridos en un Egresado de la Carrera Economía Agrícola del Departamento de Ciencias Económicas y Administrativas, de Modalidad Regular del Turno Vespertino durante el período 2007 - 2011 en la Facultad Regional Multidisciplinaria de Carazo. A través de una Estructura de Costos por Procesos que permita conocer la inversión total que realiza la UNAN - Managua en un Egresado de la FAREM - Carazo de la Carrera Economía Agrícola. Para realizar este trabajo nos auxiliamos de información proporcionada por el área de contabilidad y administración de la Facultad, la cual nos brindó información referente a la tarifa salarial de acuerdo al nivel académico de los docentes, los Costos Indirectos de Fabricación, la cantidad de estudiantes becados durante este período. Y la Unidad de Secretaría Académica nos suministró los registros de matriculados durante los cinco años que son objeto de estudio. Todos estos datos fueron clasificados de acuerdo a los elementos del costo para determinar el Costo de un Egresado de dicha Carrera. Los elementos del costo utilizados en la determinación del costo unitario de un Egresado son: Materia Prima, Mano de Obra Directa, Costos Indirectos (servicios personales y no personales, transferencias corrientes, becas académicas, servicios básicos agua y luz). Los instrumentos para la investigación, métodos y procedimientos correspondieron a entrevistas y encuestas Cualitativas-Cuantitativas. Los datos recopilados fueron sometidos a un análisis de los cuales se obtuvieron resultados importantes sobre el conocimiento de las personas interesadas sobre el Costo de un Egresado para FAREM - Carazo.

Palabras clave: costo, egresado, universidad, economía agrícola.

\section{INTRODUCCIÓN}

El presente trabajo está centrado en el interés por investigar y determinar el Costo de un Egresado de la Carrera Economía Agrícola del Departamento de Ciencias Económicas y Administrativas de la Modalidad Regular del Turno Vespertino durante el período 2007 - 2011 en la Facultad Regional Multidisciplinaria de Carazo, a través de una Estructura de Costos por Procesos que permita a los interesados identificar los elementos del costo que lo integran. Siendo una Central Costos por Procesos una innovación para la Facultad Regional Multidisciplinaria de Carazo ya que en esta registran una Contabilidad Patrimonial donde solo se refleja Ingresos contra Egresos, por lo que una Estructura de Costos sería de gran importancia para los usuarios de dicha información; permitiendo que estos tengan mayores conocimientos de la inversión que realiza la UNAN - Managua en un Egresado de dicha Carrera. Con este trabajo, se pretende hacer conciencia en los estudiantes de la Facultad Regional Multidisciplinaria de Carazo para que aprovechen la oportunidad de ser profesionales que les brinda la UNAN - Managua. La presente investigación está orientada al estudio de los costos que incurre la UNAN- FAREM - Carazo en un Egresado de la Carrera Economía Agrícola del Departamento de Ciencias Económicas y Administrativas, de la Modalidad Regular, Turno Vespertino durante el período 2007 - 2011.

\footnotetext{
${ }^{1}$ Trabajo dirigido por la licenciada Silvia Elena Carranza
} 


\section{MATERIALES Y MÉTODOS}

El tipo de estudio es descriptivo, lo cual permitió obtener información acerca de los elementos del costo que intervienen para determinar la inversión total que realiza la UNAN - FAREM - Carazo en un Egresado de la Carrera Economía Agrícola, permitiéndonos conocer datos reales sobre los costos que incurren durante el período estudiado.

Las principales fuentes de información fueron:

- Secretaria Académica de la FAREM - Carazo la cual nos facilitó el informe de matrícula durante el período estudiado y la organización de los docentes.

- Personal Administrativo, que brindó información acerca de la tarifa salarial de los docentes de acuerdo al nivel académico y los Costos Indirectos de Fabricación que intervienen de manera indirecta en la determinación del costo de un Egresado.

- Unidad de Contabilidad de la Facultad por facilitar todo lo relacionado a las becas académicas, describiendo así el número de becas entregadas y el monto de cada tipo de beca en cada semestre durante el período estudiado.

- ENACAL, por brindarnos información sobre el consumo de agua en metros cúbicos por persona y el costo en valor monetario de ese consumo; a través de una entrevista no estructurada con el Ingeniero Delegado para Carazo.

- Unión Fenosa, por su disposición al brindar datos acerca del consumo de energía eléctrica durante cada semestre.

Otras fuentes de información fueron las técnicas de investigación:

- Encuestas dirigidas a los estudiantes de Cuarto y Quinto Año de la Carrera Economía Agrícola, con el fin de saber el nivel de conocimiento que poseen respecto a la inversión que realiza la UNAN - FAREM - Carazo en la preparación de un estudiante al finalizar su carrera; siendo de gran importancia la información brindada para sustentar nuestras conclusiones y recomendaciones.

- Entrevista No Estructurada, esta técnica fue dirigida al Coordinador da la Carrera Economía Agrícola con el objetivo de que nos proporcionara información necesaria sobre las características del área, sus experiencias en el campo y las sugerencias para el logro de los objetivos de nuestra investigación.

La información recolectada a través de los procedimientos antes mencionados fue clasificada y analizada cualitativamente y cuantitativamente logrando de esta manera establecer la relación existente entre los datos proporcionados por las técnicas de recolección que utilizamos y los datos reales en las cédulas de los elementos del costo que intervienen en la inversión total que ejecuta la UNAN - FAREM - Carazo en un Egresado.

\section{RESULTADOS}

La figura 1 refleja el consolidado a lo largo de cinco años es decir a lo largo del período estudiado, en la cual se observa el comportamiento de cada uno de los aspectos que integran a los elementos del costo. Se puede observar cual elemento del costo es más alto y en qué año el costo unitario aumenta. El elemento del costo que representa mayor aumento son los salarios de planta debido a que a partir del año 2008 la mayoría de los docentes que imparten las asignaturas fueron de planta, lo que da como consecuencia que 
el costo unitario incremente por motivo al aumento de la tarifa salarial en el año 2007, 2008 y 2009 y también en el segundo semestre de cada año se paga el décimo tercer mes a los docentes de planta.

Figura 1

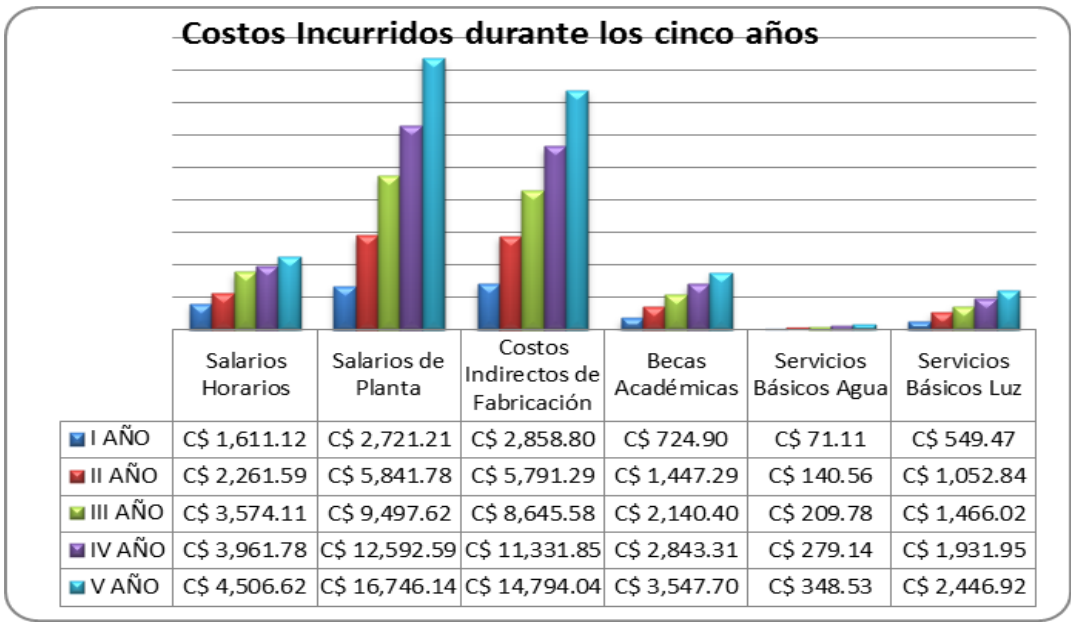

Con respecto al consolidado de los cinco años, el año que presenta el mayor aumento en relación al costo unitario es el año 2011 debido a que es el último año académico a ser evaluado y en este se vienen acumulando los costos desde el primer semestre del año 2007 a causa del aumento en la tarifa salarial, el índice de deserción que al haber menos alumnos el costo aumenta acorde a su distribución y el pago del décimo tercer mes a los docentes de planta en el segundo semestre de cada año. La figura 2 representa en términos porcentuales el índice de deserción desde el primer año hasta el último año, resultando en el último año un $75.51 \%$ lo que indica que el índice de deserción es del $25.49 \%$ con respecto al $100 \%$ que ingreso en el primer año.

La encuesta aplicada los Figura 2 Índice de Deserción Porcentual

estudiantes de Cuarto y Quinto año de la Carrera de Economía Agrícola, tenía el propósito de saber cuál es el grado de conocimiento que poseen dichos estudiantes sobre la inversión que realiza la UNAN - FAREM - Carazo para la formación de un profesional. A continuación se presentan los siguientes resultados en relación a cada pregunta:

Según la pregunta $\mathrm{n}^{\circ} 2$ se

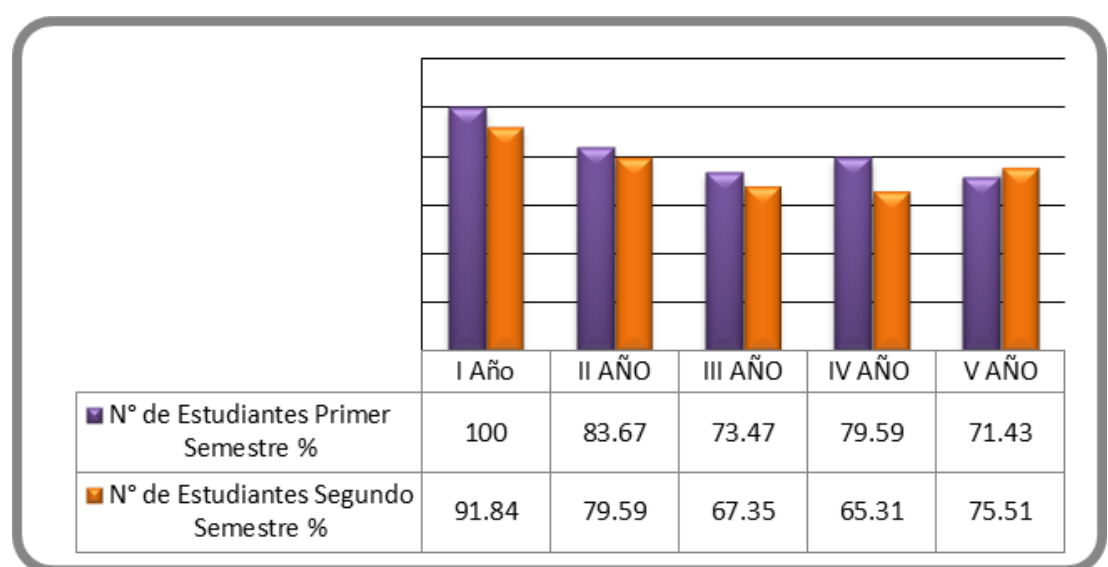
obtuvo en total que el $73.3 \%$ de los estudiantes encuestados poseen conocimiento de las carreras que oferta la FAREM - Carazo mientras que el $26.7 \%$ desconoce las carreras que ofertan. De acuerdo a la pregunta ${ }^{\circ} 3$ el $46.7 \%$ de los estudiantes encuestados tienen alguna referencia de cuanto es el número de estudiantes de la FAREM - Carazo y el 53. 3\% no tenían conocimiento alguno.

Según la pregunta $\mathrm{n}^{\circ} 4$ y $\mathrm{n}^{\circ} 5$ establece que el $86.67 \%$ del total de encuestados han sido beneficiados con cualquier tipo de beca y el $13.33 \%$ no han tenido beneficio alguno. Con respecto a la pregunta $\mathrm{n}^{\circ} 6$, se obtuvo que el $60 \%$ de los estudiantes encuestados están conscientes de que la FAREM - Carazo paga los servicios básicos, mientras que el $40 \%$ no tiene información relacionada. En relación a la pregunta $n^{\circ}$, el $26.7 \%$ de los encuestados opinan que tienen referencia con respecto al salario que devengan los docentes y 
el $73.3 \%$ mantiene abstención. Según la pregunta ${ }^{\circ} 8$, el $100 \%$ de los encuestados no poseen información en relación al salario que devenga el personal administrativo de la FAREM - Carazo. De acuerdo a la pregunta $\mathrm{n}^{\circ} 9$ el $93.3 \%$ de los encuestados tienen conocimiento de donde proviene el dinero para financiar su carrera, mientras que el $6.7 \%$ desconoce el origen de las fuentes de ingreso de la FAREM - Carazo.

Con respecto a la pregunta $\mathrm{n}^{\circ} 10$ se concluyó que el $100 \%$ de los encuestados no tienen ningún dato sobre el monto total que invierte la UNAN - FAREM - Carazo en la preparación de un estudiante al finalizar su carrera. En relación a las pregunta, $\mathrm{n}^{\circ} 11$ y n ${ }^{\circ} 12$ del total de encuestados el $53.3 \%$ estimó en valor monetario que entre $\$ 1,000.00$ y $\$ 4,000.00$ es lo que invierte la UNAN - FAREM - Carazo en un Egresado de la Carrera Economía Agrícola. De igual forma el $26.7 \%$ expresa que lo invertido por la UNAN - FAREM - Carazo en un Egresado de dicha carrera se encuentra entre los $\$ 1,800.00$ y $\$ 3,000.00$ y el $20 \%$ no dieron opinión alguna. Por lo tanto el $86.7 \%$ de los estudiantes han clasificado estos montos en un rango medio y el $13.3 \%$ de los encuestados como bajo.

De acuerdo a la entrevista que realizamos al Coordinador de la Carrera de Economía Agrícola, él opina que es de utilidad brindarle información a los estudiantes y docentes sobre la inversión que realiza la UNAN- FAREM- Carazo en un Egresado de la Carrera de Economía Agrícola, y de acuerdo a esta inversión él clasifica el costo como medio. Al igual que los estudiantes el Coordinador desconoce el salario que devengan los docentes de la FAREM - Carazo, solo conoce el salario que devenga él. Por otra parte hace mención que la UNAN - FAREM - Carazo no paga servicios básicos, sino que en su opinión solo paga el teléfono.

\section{CONCLUSIONES}

La UNAN - FAREM - Carazo contribuye a la formación científica, técnica, cultural y patriótica de los estudiantes. Realizando inversión en el capital humano para mejorar los ingresos futuros a través de la incorporación de recursos a las personas, tomando decisiones de educar al capital humano donde los individuos toman en cuenta tanto los costos presentes como los beneficios futuros. Sin embargo, esta inversión conlleva costos que son incurridos a lo largo de cinco años, para ofrecer al capital humano preparación de calidad siendo estos muchos mayores al costo de la matrícula.

De acuerdo a las encuestas practicadas a los estudiantes de la Carrera de Economía Agrícola se pudo comprobar que desconocen información sobre la inversión que realiza la UNAN - FAREM - Carazo en un Egresado.

Culminando con este trabajo hemos determinado el costo unitario real de un estudiante de la Carrera Economía Agrícola el cual asciende a C $\$ 125,936.05$ (Ciento veinticinco mil novecientos treinta y seis con 5/100).

\section{BIBLIOGRAFÍA}

Méndez, C, (1999) Metodología de la Investigación (4ª ed.).

Gayle, L. Polimeni, R. (1994). Contabilidad de Costos Conceptos y Aplicaciones para la toma de decisiones gerenciales ( $3^{\mathrm{a}}$ ed.).

Ortiz, A. (2003). Costos Indirectos. 